\title{
LAS LICENCIAS DE ESCLAVOS NEGROS A HISPANOAMÉRICA (1544-1550)
}

\author{
POR \\ ESTEBAN MIRA CABALLOS \\ Universidad de Sevilla
}

\section{PANORAMA GENERAL}

¡Qué duda cabe que el estudio de la población negra, su trata y su evolución en el Nuevo Mundo es parte fundamental para entender toda la historia del continente americano! Como es sabido, desde un primer momento se va a autorizar la entrada de negros en el Nuevo Mundo, ya que la trata negrera era algo considerado normal en la época. La esclavitud era a fines del siglo XV una institución de hondas y largas raíces en la Península Ibérica, que la sociedad del momento aceptaba como «un hecho natural" (1). Sin embargo, en los momentos previos al Descubrimiento se encontraba un tanto aletargada, pues sabemos que, por ejemplo, en Sevilla hacia 1496 había poco más de 250 esclavos (2). Con el paso de los españoles al otro lado del océano se reactivará de manera importante todo lo concerniente a la esclavitud, sobre todo a nivel particular, aunque secundados también por la Corona que pronto encontró en ella una manera de obtener fáciles beneficios.

Siglas Utilizadas:

AGI: $\quad$ Archivo General de Indias, Sevilla.

AGS: $\quad$ Archivo General de Simancas.

CODOIN: Colección de documentos inéditos relativos al descubrimiento, conquista y organización de las antiguas posesiones de América y Oceanía. Madrid, 1864-1884.

(1) Alfonso Franco Silva: La esclavitud en Sevilla y su tierra a fines de la Edad Media, Sevilla, Diputación Provincial, 1979, pág. 37.

(2) Ibidem. 
Son pues los motivos económicos los que condicionan todo lo concerniente a la trata negrera en América, ya que los españoles cada vez más necesitaban del trabajo negro dado el vertiginoso descenso de la población aborigen.

Entre 1492 y 1513 se da una etapa que Enriqueta Vila ha denominado de "aclimatación y ensayo» (3), años en los que la trata se caracteriza por la improvisación y la falta de control por parte de la Corona (4). Sabemos que ya Cristóbal Colón, en su segundo viaje, llevó algunos negros a bordo, aunque se trató de un hecho aislado pues no existía aún ninguna legislación al respecto. La Corona se pronunció finalmente en 1501, fecha en la que permitió oficialmente el paso de esclavos a las nuevas tierras recién descubiertas (5). No obstante entre 1503 y 1505 se produjo un paréntesis durante el cual el Comendador de Lares pidió al Rey que no se llevasen negros a La Española, por los continuos alzamientos que protagonizaban algunos de ellos, afirmación de la que se retractó en este último año al solicitar de los Reyes Católicos la reapertura del tráfico negrero (6).

Nuevamente, en las Instrucciones a Diego Colón de 1509 se ratificó la posibilidad de llevar negros a Indias contando siempre con la licencia de éste y con la condición imprescindible de que fuesen cristianos (7). Dos años después se permitió por primera vez, lo que se iba a convertir años después en una constante, es decir, el paso de esclavos africanos directamente sacados de las factorías portuguesas en Guinea (8). Además en este año se comenzaron a cobrar dos ducados por cada pieza llevada a Indias, cifra que con el paso de los años aumentaría considerablemente, hasta llegar a los 9 ducados que, por ejemplo, se pagaban en 1552.

Hacia 1518 se produjeron, nuevamente, varios cambios decisivos que permanecieron inmutables prácticamente a lo largo de

(3) Enriqueta VILA VILAR: "La esclavitud en el Caribe, Florida y la Luisiana: Algunos datos generales para su estudio", La influencia de España en el Caribe, Florida y la Luisiana (1500-1800), Madrid, ICI, 1983, pág. 114.

(4) Idem, págs. 114-115.

(5) Instrucción a Nicolás de Ovando, 16 septiembre 1501. CODOIN, Serie 1. a , tomo 31, págs. 13-25.

(6) Carlos Larrazabal Blanco: Los negros y la esclavitud en Santo Domingo. Santo Domingo, Postigo e hijos, editores, 1967, pág. 14.

(7) Instrucciones a Diego Colón, Valladolid, 3 mayo 1509. CODOIN. Serie 1. a, tomo 23, págs. 290-309 y tomo 31, págs. 388-409.

(8) José Antonio SAco: Historia de la esclavitud de la raza africana en el Nuevo Mundo y en especial en los paises Americo-Hispanos, tomo I, La Habana, 1938, pág. 106. 
todo el siglo XVI. Para empezar, comenzaron a ser de forma periódica los envíos desde Guinea, con lo cual se potenciaba la traida de negros bozales en detrimento de los ladinos que resultaban ser mucho más revoltosos (9). En la Junta de Procuradores de 1518 los vecinos de La Española se quejaron de lo desobedientes e insumisos que eran los esclavos que venían de Castilla, pidiendo unánimemente que tan sólo se permitiese el paso de los bozales procedentes directamente de África (10), siendo todo esto aceptado por la Corona en 1526 para posteriormente ratificarlo en 1532 (11). Es interesante ver el cambio de política tan radical con respecto a la legislación inicial, de manera que si entre 1500 y 1510 se pensó que los ladinos, por su condición de cristianos eran los idóneos para América, desde 1515 la idea cambió totalmente y se prefirieron los bozales, pues aunque no eran cristianos, tenían menos tendencia a los levantamientos.

Con respecto a estos esclavos sacados directamente de África el más común será, sin lugar a dudas, el guineano pues la legislación prohibía terminantemente el envío de esclavos berberiscos, salvo expresa licencia real. La explicación hay que buscarla en la religión islámica practicada por estos berberiscos y en la supuesta minoría de edad de los indios. En este sentido, reza un documento de 1545 que explica perfectamente esta circunstancia: "porque como sea tierra nueva no se de ocasión a que se plante entre los naturales de ella la secta de Mahoma" (12).

Otra de las medidas que se tomó es que se llevasen negras con la intención de que los esclavos formasen familias y se asentasen "porque con ésto y con el amor que tendrían a sus mujeres e hijos y con el orden del matrimonio sería cosa de mucho sosiego de ellos y se excusarían otros pecados e inconvenientes que de lo contrario se siguen" (13). Y finalmente, como otra de las medidas importantes hay que decir que se comenzó a

(9) Rolando Mellafe: Breve historia de la esclavitud en América Latina, México, 1973, pág. 31. Franco, [1] pág. 97.

(10) Junta de Procuradores en La Española, 1518. AGI, Santo Domingo 77, $\mathrm{R} 1, \mathrm{n}^{2} 1$ al 27. Puede verse también en Manuel GIMÉNEZ FERNÁNDEZ: Las Cortes en La Española de 1518. Sevilla, AUH, 1954.

(11) Real Cédula a los oficiales de la Casa de la Contratación, Sevilla 11 mayo 1526. Y Real Cédula a los oficiales de la Casa de la Contratación, Medina del Campo, 13 enero 1532. Diego de EncinAs: Cedulario Indiano, Madrid, ECH, 1945, tomo IV, pág. 384. Recopilación de leyes de los Reinos de Indias, libro IX, Título 26, ley 18.

(12) Carta de los oidores, de Santo Domingo a Su Majestad, Santo Domingo, 23 abril 1545. AGI, Santo Domingo 49, R 15, n²96.

(13) Real Cédula a los oficiales de la Casa de la Contratación, Valladolid, 28 junio 1527. AGI, Indiferente General 421, L 12, ff. 151-152. 
dar desde 1518 las grandes licencias a deudos como Laurent de Gouvenot a quien se le autorizó en este año para pasar 4.000 esclavos, o a los Welser quienes recibieron este mismo privilegio en 1528 (14).

Este es el marco legal en el que hemos de encuadrar la cuestión de la esclavitud negra en el Nuevo Mundo a lo largo prácticamente de toda la decimosexta centuria.

\section{EvOluCiÓN CUANTITATIVA}

En este panorama una de las grandes cuestiones por resolver del siglo XVI es sin duda, el estudio cuantitativo de los esclavos africanos que fueron llevados al continente americano. Desgraciadamente no existen trabajos para el siglo XVI como el que Enriqueta Vila Vilar realizó para buena parte del siglo XVII (15), debido fundamentalmente a la carencia de fuentes que posibiliten abordar un estudio similar para la primera centuria del dominio español en Indias. Por tanto ni las obras de carácter general (16), ni otros estudios más parciales han conseguido establecer una secuencia fiable y mínimamente completa de la migración forzosa de estas personas (17). Y aun para los periodos más conocides como son los de 1530 a 1540, de 1556 a 1570 y de 1597 a 1600 , en que aparecen secuencias completas de migración, éstas son excesivamente lacónicas como para permitir grandes análisis.

En medio de este limitado panorama presentamos ahora un interesante hallazgo documental, que procede del Archivo General de Simancas, en el cual se enumeran extensamente cada una de

(14) Demetrio RAMOs: "El negocio negrero de los Welser y sus habilidades monopolísticas". Revista de Historia de América, $\mathrm{n}^{\circ} 81$, México, enero-junio de 1976 (págs. 7-81), págs. 8 y ss.

(15) Enriqueta VILA VILAR: Hispanoamérica y el comercio de esclavos: Los asientos portugueses, Sevilla EEHA, 1977.

(16) G. SCELLE: Histoire politique de la traite négrière aux Indes de Castille, 2 vols. Paris, 1906 y MELLAFE [9].

(17) Entre los trabajos concretos pueden citarse los siguientes: Ramos [14], Enrique OTTE y C. RuIz-BerRuecos: "Los portugueses en la trata de los esclavos negros en las postrimerías del siglo XVI", Moneda y Crédito, 1963 (págs. 33-40). Alain Milhou: Los intentos de repoblación de la isla Española por colonias de labradores (1518-1603). Razones de un fracaso. Burdeos, 1977. Henry LAPEYRE: "La trata de negros con destino a la América Española durante los últimos años del reinado de Carlos V. 1544-1555", Cuadernos de Investigación Histórica, n 2 , Madrid, 1978 (págs. 335-339) y Lutgardo GARCía FUENTES: "La introducción de esclavos en Indias desde Sevilla en el siglo XVI" Andalucía y América én el siglo XVI, Sevilla, 1983 (págs. 249-261). 
las licencias que otorgó el Rey entre 1544 y 1550 (18). Se trata de 54 carillas de texto prieto en el que amén de relatarse todas las licencias de esclavos se especifica su número exacto, el navío en el que se transportan, el maestre de éste, el nombre del propietario de la licencia y sólo en algunos casos el destino de cada esclavo negro. Sin embargo, en los casos en que no aparece el destino éste, lo hemos podido fácilmente localizar a través de un seguimiento de los dichos mercaderes, y sobre todo de los maestres.

El documento en cuestión, responde a una real cédula que expidió el Rey en 1551 a los oficiales de la Casa de la Contratación en el cual se le solicitaba el traslado de todas las licencias de esclavos que se habían expedido entre 1544 y 1550, a efectos de conocer la renta que exactamente le debería corresponder (19). Así, el Rey pretendía cobrar todos los maravedíes que le pertenecían del pago de los derechos del almojarifazgo, desde 1544 a octubre de 1549, años en los que había figurado como almojarife un tal Pedro de Balmaseda. Éste, en nombre del Rey, había cobrado 7 reales y medio por cada una de las 9.797 piezas que se habían sacado de Guinea entre principios de 1544 y julio de 1549, a las cuales se le añadieron luego el importe del almojarifazgo de 3.610 piezas que pasaron entre agosto de 1549 y noviembre de 1550 (20).

Se trataba este almojarifazgo de un impuesto nuevo, que se había comenzado a cobrar, según parece, a partir de 1544 (21), el cual consistió hasta 1550, como ya hemos afirmado, en 7 reales y medio y desde 1550 a 1554 en 9 reales y medio (22). El propio Carlos V exponía los motivos de este nuevo impuesto de la siguiente forma:

«El trato con las Indias produce a los mercaderes que lo llevan desde Sevilla, grandes y conocidos intereses y ganancias; a sus expensas, y al amparo de la franquicias que lo fomentan,

(18) El documento en cuestión se encuentra en AGS, Consejo y Juntas de Hacienda 23, ff. 388 y ss.

(19) Real Cédula a los oficiales de la Casa de la Contratación, Cigales, 17 marzo 1551. AGS, Consejo y Juntas de Hacienda 23, ff. 390-390v.

(20) Real Cédula a los Contadores de Su Majestad, sin fecha, AGS, Consejo y Juntas de Hacienda 23, ff 388-9.

(21) M. Ulloa: La Hacienda Real de Castilla en el reinado de Felipe II, Roma, 1963, pág. 176 y ss.

(22) Real Cédula a los contadores de Su Majestad, sin fecha, AGS, Consejo y Juntas de Hacienda 23, ff. 389. 
decrece la contratación con otras partes; las necesidades que cada día se nos of recen crecen sin cesar..." (23).

Como se ve, parece evidente que también la Corona quería sacar tajada del próspero comercio indiano, aunque fuera en detrimento de los intereses particulares tanto de los mercaderes como de la familia Colón.

Son muchas las informaciones que se pueden extraer de este documento que estamos tratando del Archivo General de Simancas, cuyos datos más sobresalientes se encuentran reproducidos en el Apéndice I de este artículo. En primer lugar se puede establecer, de manera bastante fiable, el número exacto de negros que pasaron legalmente a Indias entre 1544 y 1550, lo cual es ciertamente importante ya que, como en líneas anteriores dijimos, son muy pocos los años del siglo XVI de los que se conocen la totalidad de las licencias que se dieron. Veamos las cifras a continuación:

CUADRO 1

TRATA DE NEGROS A INDIAS (1544-1550)

\begin{tabular}{ccccc}
\hline Año & Ne negros & \multicolumn{1}{c}{$\%$} & No Licencias & $\%$ \\
\hline 1544 & 660 & 5,11 & 5 & 1,71 \\
1545 & 2.169 & 16,80 & 58 & 19,86 \\
1546 & 1.710 & 13,24 & 32 & 10,95 \\
1547 & 1.392 & 10,78 & 23 & 7,87 \\
1548 & 1.992 & 15,43 & 56 & 19,17 \\
1549 & 3.217 & 24,92 & 82 & 28,08 \\
1550 & 1.768 & 13,69 & 36 & 12,32 \\
\hline TOTAL & 12.908 & 100 & 292 & $100(24)$ \\
\hline
\end{tabular}

Se puede observar en este cuadro cómo hay una cierta fluctuación interanual tanto a nivel de licencias como de cifras totales de negros, siendo 1549 el año en el que se otorgan —con

(23) Ramón CARANDE, Carlos $V$ y sus banqueros, Barcelona, Editorial Crítica, 1990, tomo 2, pág. 311.

(24) A la hora de valorar las cifras no podemos perder de vista que el año 1544 sólo aparece a partir de marzo y el de 1550 sólo abarca hasta diciembre en la lista de licencias que hemos manejado nosotros. 
diferencia- mayor número de licencias, alcanzando la cifra de 3.217 la cual es muy alta para la temprana fecha de que se trata.

Es interesante, igualmente, comprobar lo poco que se diferencian los porcentajes del número de esclavos con los del número de licencias, lo cual es bien indicativo de la cierta homogeneidad que había en el número de esclavos por licencia al menos en lo que se refiere a la media. En líneas generales el número total coincide aproximadamente con los algo más de 13.000 que calculó Lapeyre para los años completos del 1544 al 1550 (25), cifras que si bien no son tan altas como las de fines del siglo XVI durante el tiempo de los asientos portugueses, sí que vienen a corroborar unos números más elevados a las que se habían sostenido hasta hace poco más de un decenio.

No obstante, la cantidad debió ser mucho más elevada dada la alta cota de fraudes que se registra en la trata del negro. Un fraude del que la Corona era consciente, pues se muestra reiterativa en las órdenes contra estas acciones ilegales. Así, ya en 1522 advierte a los oficiales de La Española que no consientan la importación clandestina de negros, so graves penas, reiterándose la misma orden en una sobrecédula tres años después (26). En 1531 se formula una queja por parte de los mismos asentistas afirmando que a causa del gran número de esclavos que se habían pasado ilegalmente a las Indias ellos, que habían satisfecho todos los derechos, no los podían vender a precios adecuados (27). Finalmente, la Corona, dada la imposibilidad de frenar un fraude en el que estaba implicada demasiada gente, ordenó que en adelante no se consintiese y se vigilase estrechamente este tráfico pero "que no se hagan informaciones sobre cosas pasadas" (28).

Lo cierto es que el comercio esclavista tenía muchas facilidades para desfalcar; por ejemplo se solían pasar negros como grumetes en las naves, que eran vendidos una vez en las Indias (29). Pero, sobre todo, era la propia coyuntura del tráfico lo que permitía el fraude, pues había que registrarlo en Sevilla, cargarlo en África y trasladarlo a América. En una carta de la

(25) LAPEYRE, La trata [17], pág. 336.

(26) Real Cédula a los oidores de Santo Domingo, Valladolid, 13 noviembre 1522. Sobrecédula a los oidores de Santo Domingo, Madrid 5 abril 1525. AGI, Contratación 5.090, L 5 ff. 68-69.

(27) Real Cédula a los oficiales de la Casa de la Contratación, Ocaña, 16 de mayo 1531, AGI, Indiferente General 1961, L. 2, ff. 69v-71.

(28) Real Cédula a los oidores de Santo Domingo, Toledo, 8 febrero 1539. AGI, Contratación 5.090, L. 1, ff. 96-96v

(29) Véase por ejemplo respuesta a los oficiales de Sevilla, Valladolid 28 junio 1527. AGI, Indiferente General 421, L. 12, ff. 125v-126v. 
Audiencia de Santo Domingo a Su Majestad aparece perfectamente comentada esta realidad: «Que registran cien y traen diez, y otros veinte y traen cincuenta, porque cargan en las naos los negros que hallan a la sazón que por allí pasan y no los que traen registrados y porque les falta el dinero y otras veces les sobra según los negros que hallan y los precios que cuestan» (30).

Además de éstos existian otras posibles incidencias, que hacían dificultoso el control por parte de la Corona, como los naufragios o los asaltos piráticos. En este sentido, Gaspar de Torres a través de una información pudo demostrar que los 80 esclavos que registró en 1537 en Sevilla no llegaron a su destino -Santo Domingo- porque la nave naufragó en los ríos de Guinea (31). De la misma forma demostró Rodrigo de Dueñas que en 1545 fue asaltada su embarcación por piratas y robadas sus licencias de negros (32).

Todas estas circunstancias daban lugar a un fácil y poco arriesgado engaño, de manera que en 1537, por citar un ejemplo llamativo, se descubrió que Alonso de Idiáquez y Diego de Zárate, con una licencia de cien esclavos habían pasado el dicho número por tres veces, por no habérsele rasgado la licencia (33).

Pero además del contrabando hay que tener en cuenta otro factor, que contribuye a pensar en un número mucho mayor de esclavos llevados a Indias. En las licencias que se enumeran en la relación de los almojarifes sólo aparecen aquellos esclavos que fueron sacados directamente de Guinea, pero en ningún caso los abundantes mulatos y esclavas "blancas» que los pasajeros a Indias solían llevar "para remedio de sus casas y personas". Nosotros hemos localizado entre 1540 y 1550 unas veinticinco licencias que suman más del medio centenar de esclavos, la mayoría de ellos mulatos de servicio o mulatas "cocineras» (34).

Todo lo señalado hasta el momento, tanto por la cuestión del fraude como por los datos que no aparecen registrados en el

(30) Carta de la Audiencia de Santo Domingo a su Majestad, Santo Domingo, 20 octubre 1528. AGI, Santo Domingo 49, R 9, n 63.

(31) Información hecha a petición de Gaspar de Torres, Sevilla, 19 agosto 1538. AGI, Indiferente General 1.205, $n^{2} 56$.

(32) Información hecha a petición de Rodrigo de Dueñas, 12 agosto 1545. AGI, Indiferente General 1.207, $\mathrm{n}^{\circ} 41$.

(33) Real Cédula a los alcaldes ordinarios de Puerto Rico, Valladolid, 17 febrero 1537. AGI, Contratación 5.090, L 1, ff. 24v-25v.

(34) Las licencias que mencionamos las hemos recopilado del Archivo General de Indias, secciones Indiferente General 1.207. 1963 y 1964 y Audiencia de Santo Domingo 868, libros 1 y 2. En la Sección de Indiferente General se conservan muchas de estas licencias para el resto de los años. 
documento que ahora analizamos, son ciertamente indicativos de que el número de esclavos llevados a las Indias fue ya por estas fechas muy elevado. Cantidades que para los años 1544 a 1550 son incluso más altas que las señaladas por García Fuentes para el periodo de 1530 a 1539, pues mientras éste calculaba una media de 610 esclavos anuales (35) nosotros hemos contabilizado 1.844. Sin duda, esto hay que explicarlo tanto en función de una mayor demanda desde el otro lado del Océano, como por las propias necesidades económicas de la Corona que le impulsó a buscar nuevos ingresos. Así, como es sabido, en la década de los cuarenta, y especialmente desde 1544, la Corona española entró en unos momentos que Carande denominó de «incertidumbre económica", situación a la que se llegó sobre todo por la sangría que estaba suponiendo la guerra en Alemania (36). Es en estas circunstancias, cuando la Corona se decidió a crear el nuevo almojarifazgo que se extendía a la trata negrera y a fomentar, por encima de todo, el envío de negros a las Indias como forma de obtener unos ingresos inmediatos.

En lo que concierne al sexo de estos esclavos es poco lo que podemos decir a la luz de estas licencias que presentamos. En la mayoría de las licencias, y según aparece en el texto, se estipulaba que un tercio de las piezas fuesen hembras, cantidad que venía siendo la norma desde hacía varias décadas. Aunque en otras ocasiones la Corona decía que de setenta fuesen veinte hembras o de sesenta, diez (37). A no dudarlo, esta medida de incluir hembras entre los envíos de negros respondía al interés de la Corona porque los negros se casasen, para evitar así los peligrosos alzamientos que de continuo llevaban a cabo. En este sentido, la Junta de Procuradores de la isla Fernandina reunida en 1528 fue contundente al solicitar del Rey que no se consintiese el paso de esclavos negros a esa isla si no venían hembras en igual número, "para que casándose vivan en más paz y sosiego» (38). De manera que la Corona proveyó que en un plazo máximo de quince meses todos los poseedores de esclavos los casasen, so pena de perderlos, plazo que tuvo que ser ampliado a dos años por la

(35) García Fulntres [17], 57, Cuadro no ${ }^{-}$.

(36) CaRANDE [23], tomo III, págs. 256 y ss.

(37) Real Cédula a los oficiales de la Casa de la Contratación, Valladolid, 30 junio 1527. AGI, Indiferente General 421, L 12, ff. 128-129v.

(38) Memorial dirigido al Rey por la Junta de Procuradores de la isla Fernandina, Santiago 17 marzo 1528. AGI, Patronato 178, R 10. 
imposibilidad física de cumplir la cédula en tan corto espacio de tiempo (39).

Otro de los puntos que nos parece importante tratar, y que la documentación que hemos manejado nos lo permite, con un cierto grado de fiabilidad, es el destino de estos esclavos en las fechas que ahora estudiamos, lo cual aparece reflejado en el cuadro que viene a continuación:

Cuadro 2

DESTINO DE LOS ESCLAVOS (1544-1550)

\begin{tabular}{lrr}
\hline Destino & Número & Porcentaje \\
\hline Nueva España & 3.467 & 47,31 \\
La Española & 1.175 & 16,03 \\
Tierra Firme ( $\left(^{\circ}\right)$ & 1.869 & 25,50 \\
Popayan & 349 & 4,76 \\
Puerto Rico & 257 & 3,50 \\
Honduras & 208 & 2,83 \\
Rio de la Plata & 3 & 0,04 \\
\hline TOTALES & 7.328 & 100 \\
\hline
\end{tabular}

(*) En la documentación aparece en la mayoría de las ocasiones la distinción entre Nombre de Dios y Tierra Firme, no obstante habida cuenta de que aquélla era un punto concreto dentro de ésta hemos preferido sumarlas juntas. El número de negros reseñado a Tierra Firme era de 1.111 , mientras que los que estaban destinados a Nombre de Dios alcanzaron la cifra de 758 .

Los números que aparecen en el Cuadro 2 son sólo aproximativos ya que de las 12.908 licencias localizadas sólo conocemos el destino, de manera fiable, de 7.328, es decir, de algo más de la mitad de ellas. Es por este motivo por el que deben ser tomadas con gran cautela. El destino mayoritario que aparece es el de Nueva España, que ya para la década de los cuarenta estaba perfectamente asentada la colonización y en franca expansión. En segundo lugar, destaca Tierra Firme a donde se llevan más

(39) Real Cédula a los oficiales de la isla Fernandina, 16 marzo 1528. AGI, Santo Domingo 9, R III, $n^{\circ} 1$. 
del $25 \%$ de los negros, lo cual hay que explicarlo en función de su temprano poblamiento, pues no debemos olvidar que la ciudad de Nombre de Dios se había fundado por Diego de Nicuesa en 1510. Casi 4/5 partes de las licencias tienen como destino por Tierra Firme y Nueva España, según se deduce de la suma de los porcentajes referidos a Tierra Firme, Nombre de Dios y Nueva España.

Es de destacar seguidamente el alto número de licencias que tienen como destino la isla Española teniendo en cuenta que ya por aquel entonces había perdido su hegemonía, y se encontraba poco poblada. Sin embargo, en aquella isla, dado el rápido exterminio de la población aborigen, la mano de obra negra resultaba imprescindible, tanto para los hatos y haciendas como, sobre todo, para los 26 ingenios que por aquel entonces existían y producían azúcar en la isla antillana (40).

En lo que respecta a las bajas cifras referidas a Popayán, Puerto Rico, Honduras y Río de la Plata hay que explicarlas en función de su condición, en estos momentos, de provincias marginales. El Río de la Plata, por ejemplo, con tan sólo un envío de tres negros en estos seis años, delata bien la reducidísima importancia que en este siglo XVI iba a tener tal provincia. El Perú por su parte no aparece en ningún caso como lugar de destino, sin duda porque la conquista se había llevado a cabo recientemente por lo que, por un lado había gran disponibilidad de mano de obra indígena, y por otro aún estaba en fase de afianzamiento la colonización.

Finalmente, Puerto Rico con tan sólo un 3,5 por ciento, hay que decir que junto al resto de las islas antillanas se encontraba, como ya se ha dicho, desde hacia algunas décadas, en franca decadencia. Los desastres naturales ocurridos en 1530 habían dejado la isla en una situación muy precaria. Los vecinos se quejaban de no poder comprar esclavos a los precios -60 ó 70 castellanos- que los mercaderes solían pedir por ellos (41). En lo que atañe a la isla de Cuba, ni tan siquiera aparece como lugar de destino, lo cual denota el despoblamiento que desde la década de los treinta había sufrido la isla, y las carencias económicas de una población que se seguía aferrando a los servicios de una población india en trance de desaparecer.

(40) En este sentido puede verse la obra de Justo Moreno Del Río: Los inicios de la agricultura europea en el Nuevo Mundo, Sevilla, Gráficas del Guadalquivir, 1991, págs. 308 y ss.

(41) Carta de los oficiales de la isla de San Juan a Su Majestad, Puerto Rico, 8 septiembre 1530. AGI, Santo Domingo 166, ff. 104-105.

R. I., 1994, n²01 


\section{LOS TRATANTES DE ESCLAVOS}

A continuación vamos a analizar un último aspecto, el de las personas que obtenian las licencias para llevar esclavos al Nuevo Mundo, siendo en este caso la fiabilidad casi total dado lo completa que es la fuente en lo concerniente a este punto. Veamos el Cuadro 3 en el que aparecen los principales tenentes de licencias:

\section{CuAdro 3}

PRINCIPALES TRATANTES DE ESCLAVOS (1544-1550)

\begin{tabular}{lrr}
\hline \multicolumn{1}{c}{ Nombre } & Número & Porcentaje \\
\hline Gaspar de Torres & 2.032 & 15,74 \\
Alonso de Paredes & 1.694 & 13,12 \\
Gonzalo y Gaspar Jorge & 1.322 & 10,24 \\
Hernando de Jerez y Herederos & 1.055 & 8,17 \\
Diego Caballero & 996 & 7,71 \\
Alonso Martínez & 679 & 5,26 \\
Melchor de Carrión & 430 & 3,33 \\
Francisco Núñez Pérez & 340 & 2,63 \\
Rodrigo Bazo & 209 & 1,61 \\
Resto & 4.153 & 32,17 \\
\hline TOTALES & 12.908 & 100 \\
\hline
\end{tabular}

De este cuadro se pueden extraer muchos datos interesantes para el estudio de la trata negrera que ahora estudiamos. Primero, es fácilmente observable la gran concentración de las licencias en unas pocas manos, pues tan sólo nueve personas controlaban el 70 por ciento de todas las concesiones. La mayoría de estos asentistas eran bien mercaderes, bien banqueros, o bien ambas cosas a la vez. Muchos, que empezaron como mercaderes de negros, terminaron formando parte de la nobleza a través de su ingreso en los cabildos de sus ciudades y villas. Este era el caso del mariscal Diego Caballero, veinticuatro de la ciudad de Sevilla, y del jurado Gaspar de Torres, así como de otros comerciantes de esclavos con menor número de licencias como era el caso del jurado Alonso de la Barrera o de Rodrigo de Dueñas (ver Apén- 
dice), banquero y regidor de Medina del Campo (42). Todos ellos habían obtenido gran número de licencias de esclavos, alcanzando con sus negocios el status de nobles.

El cabildo era el medio más rápido para ascender socialmente al rango de noble. Sin duda, era la vía más fácil para borrar el pasado plebeyo de muchos de estos enriquecidos ya que junto a la adquisición de una regiduría o juraduría se procedía a usu eliminación de las listas de pecheros que están pregonando su condición original» (43). Sin embargo, una vez ennoblecidos no renegaban de los oficios con los que habían ascendido social y económicamente, sino que continuaban con sus labores de mercaderes y prestamistas. En este sentido Domínguez Ortiz llega a decir lo siguiente refiriéndose en concreto a Sevilla: «El noble sevillano no miraba las actividades mercantiles o bancarias con la virtuosa indignación que sus congéneres del interior de España; mas tampoco puede decirse que sintieran auténtica vocación por los negocios; más bien fue una necesidad abrazada como mal menor, un expediente para eludir la decadencia económica...» (44). Por otra parte, el poder económico alcanzado por estos ricos mercaderes le hacían ser blancos muy deseados de la nobleza sevillana, quien a través de la vía matrimonial, lograban entroncar a sus hijos y descendientes con estos potentados personajes, beneficiándose ambos grupos de esta fusión, al conseguir unos el status nobiliario y otros la riqueza necesaria para mantenerlo. Morgado en su Historia de Sevilla nos cuenta la vida de un mercader que casó a su hija con un noble dotándola con la astronómica cifra de 240.000 ducados (45).

En cuanto a la vida de cada uno de ellos no es demasiado lo que sabemos. En lo que toca a Gaspar de Torres, que era uno de los grandes comerciantes de esclavos, a juzgar por el número de licencias que recibió de la Corona, sabemos que llegó a ser jurado en el cabildo de Sevilla, y que desarrolló en él una intensa labor política (46). Al comercio negrero se venía dedicando desde hacía años, pues ya en 1537 había comprado a Cristóbal Francis-

(42) Información hecha a petición de Rodrigo de Dueñas, 12 agosto 1545. AGI, Indiferente General 1.207, $\mathrm{n}^{\circ} 41$.

(43) Blanca Morell Peguero: Mercaderes y artesanos en la Sevilla del Descubrimiento, Sevilla, Diputación Provincial, 1968, pág. 184.

(44). Antonio Domínguez Ortiz: Orto y ocaso de Sevilla. Sevilla, Diputación Provincial, 1991, pág. 87.

(45) Citado por Antonio García-Baouero: Andalucia y la Carrera de Indias 1492-1824), Barcelona, Biblioteca de Cultura Andaluza, 1986, pág. 59.

(46) Infomación hecha por Gaspar de Torres, Sevilla 19 agosto 1538. AGI, Indiferente General 1.205, $\mathrm{n}^{\mathrm{Q}} 56$. 
quin y Diego Núñez 1.500 licencias de esclavos, que éstos habían obtenido del Rey tras desembolsar 9.750 ducados en conceptos de derechos e impuestos (47).

Respecto a Alonso de Paredes sabemos que era un tejedor de telares de Segovia (48), que probó fortuna con el comercio americano, obteniendo enormes riquezas. Los hermanos Jorge, por su parte, formaron un tándem que obtuvo grandes beneficios en el comercio con el Nuevo Mundo, alcanzando especial fuerza en la década de los cuarenta, continuando tal actividad hasta su desafortunada quiebra en 1567 (49).

Ahora bien, la importancia económica, social y política de estos tratantes de esclavos no se circunscribió exclusivamente a los grandes comerciantes de negros, pues, como puede apreciarse en el Apéndice que acompaña a este trabajo, entre los que podríamos denominar medianos y pequeños comerciantes aparecen individuos que también alcanzaron no sólo fama, sino, sobre todo, riqueza. El ejemplo más claro de todos ellos tal vez sea el del famoso Antonio Corzo, auténtico patriarca y fundador de la dinastía de los Corzo, que en unas pocas décadas se convirtió en una de las familias más ricas de toda Sevilla. Este primer Corzo no era más que uno de tantos otros extranjeros que a lo largo del siglo XVI se afincaron en Sevilla atraídos por las enormes posibilidades que este puerto y llave de las Indias ofrecía. Ya en 1525 lo encontramos comerciando con las Indias, e instalado - hacia 1544 en el barrio de Triana (50). En este caso ni siquiera fue necesario esperar a la segunda generación para ennoblecerse, pues el mismo Antonio Corzo, que procedía de una baja extracción social, llegó en su propia vida a «tener trato con gente principal" y a alegar hidalguía y nobleza (51).

Junto a estos mercaderes que estamos estudiando, figuraban también gran número de banqueros que asistieron a Carlos $\mathrm{V}$ con préstamos en los momentos de crisis, en cuya compensación debieron recibir sin duda las licencias de esclavos. Entre los banqueros que registramos con licencias de esclavos se pueden citar los siguientes: los hermanos Alonso y Fernando de Illescas, Alonso Martínez, Jerónimo Castaño, Diego Alemán, Alonso de

(47) Ibidem.

(48) CaRAnde [23] tomo I, pág. 507.

(49) Francisco Morales PADRón: La ciudad del Quinientos, Utrera, Universidad de Sevilla, 1977, pág. 84.

(50) Enriqueta VILA VILAR: Los Corzo y los Mañara: Tipos y arquetipos del mercader con América, Sevilla, EEHA, 1991, págs. 44-45.

(51) Ibidem. 
Baeza y sobre todo a Rodrigo de Dueñas. Respecto a este último sabemos que el 10 de mayo de 1546 prestó al Rey la friolera cifra de 62.000 escudos, que no recuperó hasta octubre de 1548, eso sí con un incremento del 12 por ciento anual (52).

En definitiva se trata de grandes banqueros y comerciantes, que incluso en una sola generación accedieron a la nobleza, o en cualquier caso se mezclaron matrimonialmente con ella para en una generación posterior integrarse plenamente.

\section{Las Compañías en la Trata Negrera}

Por último, otro de los aspectos que nos ha parecido interesante destacar en este trabajo es el peso que tienen, ya en estos momentos, las compañías, en lo concerniente a la trata negrera en las fechas que ahora estudiamos. Veamos el Cuadro $n^{2} 4$ :

\section{Cuadro 4}

COMPAÑíAS EN LA TRATA NEGRERA (1544-1550)

\begin{tabular}{lcc}
\multicolumn{1}{c}{ Miembros } & Licencia & $\%$ \\
\hline $\begin{array}{l}\text { Andrés de Paredes, Melchor Barreton y Alonso } \\
\text { de la Barrera }\end{array}$ & 1.524 & 38,78 \\
$\begin{array}{l}\text { Gonzalo y Gaspar Jorge } \\
\text { Hernando de Jerez, Juan Núñez, Gonzalo Ruiz, }\end{array}$ & 1.028 & 26,16 \\
$\quad$ Juan Martínez y Rodrigo Bazo (*) & 844 & 21,48 \\
Melchor de Carrión, García de Vascones y Diego & 375 & 9,54 \\
$\quad$ Caballero & 128 & 3,25 \\
Juan Pinto y Alonso Martínez & 18 & 0,45 \\
Pero López, Alonso Díaz y Rodrigo de Torres & 12 & 0,30 \\
Sancho Caballero y Diego Alemán & 3.929 & 100 \\
\hline$\quad$ TOTALES & & \\
\hline
\end{tabular}

(*) En este caso no es que fuera una compañía entre cinco sino que aparecen indiferentemente unos con otros asociados, en cada licencia.

(52) Carande, [23], tomo III, pág. 278. 
Las cifras son suficientemente significativas, en lo que respecta a la importancia de las compañías en el tráfico negrero, pues es fácil deducir de los datos de este cuadro que un tercio de las licencias las ostentaban de dos o más miembros. No obstante pensamos que la trata a través de compañías sería mayor de lo que se refleja en la documentación, pues conocemos casos en los que sólo figura la persona que diligencia el permiso de importación en la Casa de la Contratación como jefe de la compañía, extendiéndose sin embargo la merced al resto de los miembros de la misma. Esta tendencia a juntarse los mercaderes en compañías hay que entenderla en función de la escasa disponibilidad de capitales en Sevilla. Se trataba, en la mayoría de los casos, de asociaciones de molde medieval como la "commenda" o la "societas", aunque impropiamente se les conozca como compañías. En la primera los contratantes se asocian para compartir riesgos y ganancias en un solo viaje: uno o varios de ellos ponían el capital y se quedaba en tierra y otro contribuía con la tercia parte menos de capital y capitaneaba la expedición, repartiéndose por igual los beneficios al regreso, y disolviendo posteriormente la compañía. El otro tipo de asociación tiene un carácter más firme, pues se constituye no ya para un solo viaje, sino para un espacio de tiempo que solía ser, al menos, de dos años (53).

En cuanto a la frecuencia con que aparecen las compañías con respecto al número de miembros, es en 35 ocasiones de dos miembros y en las 17 restantes de tres. En ningún caso aparecen compañías formadas por más de tres personas.

(53) Jaime Vicens Vives: Historia de España y América social y económica, tomo III, Barcelona, Ed. Vicens-Vives, 1985, págs. 410 y ss. 


\section{APÉNDICE}

\section{LICENCIAS DE IMPORTACIÓN DE ESCLAVOS NEGROS A HISPANOAMÉRICA (1544-1550)}

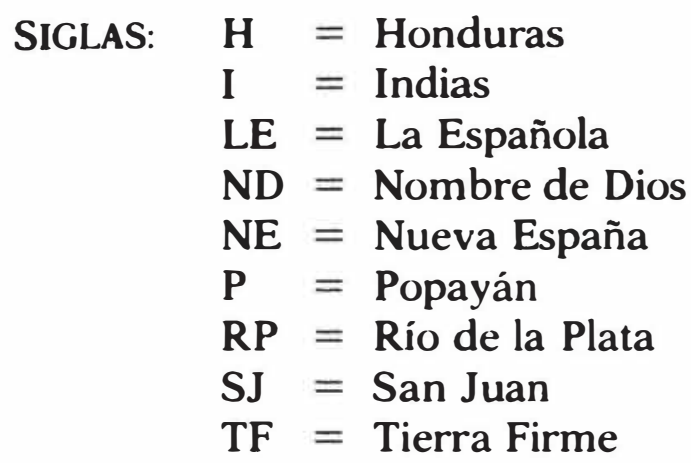

Fecha

Asentista

Maestre

№ de Destino esclavos

1544

11-III Rodrigo de Dueñas

27-IX Jerónimo de Herrera

27-IX A. de Paredes, M.B. y A.B.

27-IX A. Paredes, M. Barreto y

A. de la Barrera

22-XI Nicolao Marín

1545

5-III Francisco Carrasco

7-III Gaspar de Torres

11-III Jácome de Rodas

11-III Antonio Corzo

15-III Juan de Oña

7-IV Nicolao Blanco

7-IV Alonso de Illescas

10-IV Rodrigo de Illescas

16-IV M. Carrión, G. de Vascones y el Mariscal D. Caballero

17-IV M. Carrión, G. de Vascones y Diego Caballero

17-IV Gaspar de Torres

17-IV El M. Diego Caballero
Antonio Díaz

Antonio Díaz

Antonio Díaz

Antonio Díaz

Antonio Díaz

Sebast. González

Sebast. González

60

146

G. de Herrera

130 ND

$124 \mathrm{NE}$

200 NE

$\begin{array}{lll}\text { G. de Herrera } & 124 & \text { NE } \\ \text { Alonso Marin } & 200 & \text { NE }\end{array}$

Sebast. González $\quad 45 \quad P$

Sebast. González $11 \quad P$

Sebast. González $30 \quad P$

Sebast. González $8 \quad$ P 
Fecha

Asentista

Maestre

$N^{Q}$ de Destino

esclavos

17-IV Rodrigo de Illescas

Alonso de la Barrera

10-V Gonzalo y Gaspar Jorge

10-V Gonzalo y Gaspar Jorge

12-V Sancho Caballero y Diego Alemán

16-V Francisco Ruiz

18-IV A. Paredes, M. Barretón y Alonso de la Barrera

18-V Gonzalo y Gaspar Jorge

18-V Gonzalo y Gaspar Jorge

18-V Bartolomé de Niza

5-VI García Díaz

9-VI M. Carrión y G. Vascones

9-VI M. Carrión y G. de Vascones

13-VI M. Carrión y G. Vascones

13-VI M. Carrión y G. de Vascones

15-VI M. Carrion y G. de Vascones

15-VI Nicolao de Marín

15-VI Francisco Sánchez

16-VI Rodrigo Bazo

17-VI Andrés de Lantadilla

17-VI Diego Pérez

16-VII Rodrigo Bazo

17-VII Rodrigo Bazo

17-VII Bernaldo Pérez

20-VII Rodrigo Bazo

28-VII Juan Núñez

29-VII Juan Núñez

30-VII A. Paredes, M. Barretón y Alonso de la Barrera

4-VIII Nicolao de Marín

3-VIII Gaspar de Torres

12-VIII Nicolao de Marín

30-VIII Jerónimo Castaño

31-VIII Octaviano de Negrón

31-VIII Diego Martin

31-VIII Gerónimo Castaño

31-VIII Octaviano de Negrón

31-VIII Gerónimo Castaño

2-IX Clemente de Santana

$\begin{array}{lrl}\text { Sebast. González } & 10 & \text { P } \\ \text { Gaspar Esteban } & 18 & \text { LE } \\ \text { Gaspar Esteban } & 14 & \text { LE } \\ \text { Gaspar Esteban } & 12 & \text { LE } \\ \text { Gaspar Esteban } & 2 & \text { LE } \\ & & \\ \text { G. de Herrera } & 250 & \text { I y TF } \\ \text { Gaspar Esteban } & 126 & \text { LE } \\ \text { Gaspar Esteban } & 14 & \text { LE } \\ & 3 & \text { LE } \\ & 3 & \text { LE } \\ \text { Diego López } & 5 & \text { L.E } \\ \text { Diego López } & 50 & \text { LE } \\ \text { Diego López } & 40 & \text { LE } \\ \text { Diego López } & 13 & \text { LE } \\ \text { Diego López } & 47 & \text { LE } \\ \text { ? } & 25 & \text { I y TF } \\ \text { Diego López } & 4 & \text { LE } \\ \text { Francisco García } & 40 & \text { ND } \\ \text { Diego López } & 10 & \text { LE } \\ \text { Diego López } & 6 & \text { LE } \\ \text { Francisco García } & 30 & \text { ND } \\ \text { Francisco García } & 6 & \text { ND } \\ \text { Diego Martín } & 9 & ? \\ \text { Francisco García } & 6 & \text { ND } \\ \text { Juan de Seva } & 40 & ? \\ \text { Juan de Seva } & 40 & ? \\ & & \end{array}$

G. de Herrera $\quad 120 \quad$ I y NE

Francisco Marín 125 NE

? 220 LE?

Francisco Marín 10 NE

Diego Martin 130 ?

Diego Martín 2 ?

Dicgo Martín 6 ?

Diego Martín 1 ?

Diego Martín 2 ?

Diego Martín 4 ?

Francisco de Marín 2 ? 


\begin{tabular}{|c|c|c|c|c|}
\hline Fecha & Asentista & Maestre & $\begin{array}{c}\mathbf{N}^{9} \text { de } \\
\text { esclavos }\end{array}$ & Destino \\
\hline
\end{tabular}

$\begin{array}{ll}\text { 7-IX } & \text { H. Sánchez de la Barrera } \\ \text { 11-IX } & \text { Diego Hurtado } \\ \text { 30-IX } & \text { Nicolao Fresco Ragio } \\ \text { 26-X } & \begin{array}{l}\text { A. de Paredes, Melchor Barreto } \\ \text { y A. de la Barrera }\end{array} \\ \text { 4-XI } & \text { Mariscal Diego Caballero } \\ \text { 24-XI } & \text { Mariscal Diego Caballero } \\ \text { 24-XI } & \text { Mariscal Diego Caballero } \\ \text { 24-XI } & \text { Mariscal Diego Caballero } \\ \text { 24-XI } & \text { Mariscal Diego Caballero }\end{array}$

$\begin{array}{lrl}\text { Amador González } & 43 & \text { SJ } \\ \text { Amador González } & 17 & \text { SJ } \\ \text { Agustín de Abrego } & 140 & ? \\ & & \\ \text { Antonio Hernández } & 100 & ? \\ & 50 & ? \\ \text { Francisco Pavón } & 80 & \text { P } \\ \text { Francisco Pavón } & 50 & \text { P } \\ \text { Alonso Martín } & 25 & ? \\ \text { Alonso Martín } & 70 & ?\end{array}$

1546

$\begin{array}{ll}\text { 9-I } & \text { Gaspar de Torres } \\ \text { 8-III } & \text { Jurado Gaspar de Torres } \\ \text { 20-IV } & \begin{array}{l}\text { Mariscal Diego Caballero, M. Ca- } \\ \text { rrión y G. Vascones }\end{array} \\ \text { 10-VI } & \text { Sebastián de Palavesin } \\ \text { 10-VI } & \text { Sebastián Palavesin } \\ \text { 10-VI } & \text { Sebastián Palavesin } \\ \text { 16-VI } & \text { Juan de la Barrera el Mozo } \\ \text { 25-VI } & \text { Martín López } \\ \text { 3-VII } & \text { Jurado Gaspar de Torres } \\ \text { 10-VII } & \text { A. de Paredes, M. Barreto y } \\ & \text { Alonso de la Barrera } \\ \text { 10-VII } & \text { Gerónimo de Herrera } \\ \text { 10-VII } & \text { A. Paredes, M. Barreto y Alonso } \\ & \text { de la Barrera } \\ \text { 9-VIII } & \text { Jurado Francisco Ruiz } \\ \text { 20-IX } & \text { Pero Sánchez Fuentes } \\ \text { 20-IX } & \text { Pero Sánchez Fuentes } \\ \text { 20-IX } & \text { Pero Sánchez Fuentes } \\ \text { 9-X } & \text { Jurado Gaspar de Torres } \\ \text { 15-X } & \text { Mariscal Diego Caballero } \\ \text { 15-X } & \text { Mariscal Diego Caballero } \\ \text { 15-X } & \text { Mariscal Diego Caballero } \\ \text { 15-X } & \text { Mariscal Diego Caballero } \\ \text { 16-X } & \text { Hernando de Jerez } \\ \text { 20-X } & \text { Francisco Rucho } \\ \text { 20-X } & \text { Melchor de Carrión } \\ \text { 22-X } & \text { Alonso Báez }\end{array}$

$\begin{array}{lrl}? & 80 & ? \\ ? & 300 & ? \\ ? & 25 & ? \\ \text { Gaspar Salvago } & 35 & \text { LE } \\ \text { Gaspar Salvago } & 5 & \text { LE } \\ \text { Gaspar Salvago } & 100 & \text { LE } \\ \text { Gaspar Salvago } & 25 & \text { LE } \\ \text { Martín López } & 8 & ? \\ ? & 180 & ?\end{array}$

G. de Herrera $30 \quad$ ND

G. de Herrera $30 \quad$ ND

G. de Herrera $200 \quad$ ND

? 49 ?

Juan García $20 \quad$ ND

Juan de Almansa 9 ND

Juan García $20 \quad$ ?

Sebast. Pérez $\quad 80 \quad \mathrm{H}$

Alonso Vaez $\quad 39$ ?

Alonso Vaez 8 ?

Alonso Vaez 2 ?

Alonso Vaez 10 ?

Alonso Vaez 25 ?

Rodrigo de Leiva $12 \quad$ I

Alonso Vaez 30 ?

Alonso Vaez. 3 ?

R. I., 1994, $\mathrm{n}^{9} 201$ 


\begin{tabular}{|c|c|c|c|c|}
\hline Fecha & Asentista & Maestre & $\begin{array}{c}\mathrm{N}^{Q} \text { de } \\
\text { esclavos }\end{array}$ & Destino \\
\hline
\end{tabular}

15-XI Juan Rodriguez del Pozo

15-XI Gonzalo y Gaspar Jorge

16-XI Mariscal Diego Caballero, M. Carrión y G. Vascones

22-XII Jurado Gaspar de Torres

20-XII Jurado Gaspar de Torres

22-XII Jurado Gaspar de Torres

$\begin{array}{lrl}\begin{array}{lr}\text { Gaspar Esteban } \\ \text { Gaspar Esteban }\end{array} & 6 & \text { LE ? } \\ \text { Gaspar Esteban ? } & 39 & \text { LE ? } \\ ? & 80 & ? \\ ? & 15 & ? \\ ? & 25 & ?\end{array}$

1547

5-III Fco. Núñez de Illescas

5-III Juan Canelas

5-III Bartolomé de Vizcaya

14-III Gonzalo y Gaspar Jorge

20-IV Baltasar Pérez

20-IV D. Caballero, M. Carrión y García de Vascones

20-IV Mariscal Diego Caballero

20-IV Mariscal Diego Caballero

20-IV Francisco Ponce

24-IV A. de Paredes, B. Barreto y Alonso de la Barrera

5-V Gaspar de Torres

6-X Juan de Almansa

16-XI D. Caballero, M. Carrión y García de Vascones

16-XI D. Caballero, M. de Carrión y García de Vascones

19-VIII Bartolomé Gómez

3-IX Gaspar de Torres

25-X Luis Sánchez

25-X Luis Sánchez

2-XI Gonzalo y Gaspar Jorge

3-XI Rodrigo Pérez

11-XI Juan Núñez y $H$. de Jerez

14-XI Juan Núñez y $\mathrm{H}$. de Jerez, su hermano

2-XII Andrés de Paredes, Melchor Barreto y A. de la Barrera
Juan Enriquez

Juan Enriquez

Juan Enriquez

Vicente Díaz

Domingo Alonso

Domingo Alonso

Domingo Alonso

Domingo Alonso

Domingo Alonso

$?$

?

Juan de Almansa

Vicente Díez

Vicente Díez?

Juan de Almansa

?

Juan de Almansa

Juan de Almansa

Esteban Darea

Esteban Darea

Blas Alonso

Blas Alonso

?

$\begin{array}{rl}100 & \text { NE } \\ 119 & \text { NE } \\ 2 & \text { NE } \\ 20 & ? \\ 1 & P \\ & \\ 25 & P \\ 54 & P \\ 12 & P \\ 4 & P\end{array}$

100 ?

150 ?

3 ND?

39 ?

$\begin{array}{rl}61 & ? \\ 2 & \text { ND ? } \\ 150 & ? \\ 2 & \text { ND ? } \\ 87 & \text { ND } \\ 150 & ? \\ 10 & ? \\ 120 & \text { NE }\end{array}$

$15 \quad \mathrm{NE}$

$400 ?$ 


\begin{tabular}{|c|c|c|c|c|}
\hline Fecha & Asentista & Maestre & $\begin{array}{c}\mathrm{N}^{\mathbf{2}} \text { de } \\
\text { esclavos }\end{array}$ & Destino \\
\hline
\end{tabular}

1548

12-I Jerónimo Castaño

12-I Jerónimo Castaño

17-II A. de Paredes, Melchor Barreto y A. de la Barrera

7-III Gonzalo Jorge y Juan Díaz Gibraleón, su yerno

7-III Gonzalo y Gaspar Jorge

7-III Rodrigo Pérez

14-III Juan Núñez y Gonzalo Ruiz

14-III J. Pinto y Alonso Martínez

14-III Alonso Martínez

14-III Alonso Díaz y Juan Pinto

14-III J. Pinto y Rgo. de Torres

14-IV Gaspar de Torres

7-VI Gaspar de Torres

7-VI Gaspar de Torres

7-VI Gaspar de Torres

7-VI Gaspar de Torres

16-VI Pero Ruiz

16-VI Juan Martínez

11-VII Bartolomé de Vizcaya

30-VIII Domingo de Azpeitia

8-VIII Juan López

8-VIII P. López y A. Díaz de L.

8-VIII Juan López Piloto

8-VIII Diego Pérez

8-VIII Rodrigo de Torres

9.VIII Gonzalo Ruiz de Huelva y Juan Martínez

8-VIII J. Pinto y A. Martínez

8-VIII Gaspar de Torres

23-VIII Juan Enríquez

23-VIII Hdo. de Jerez y J. Núñez

23-VIII Pedro de la Algaba

27-VIII Cebrian de Caritate

27-VIII Pedro de la Algaba

30-VIII Domingo de Azpeitia

31-VIII Jurado J. de la Barrera

31-VIII Rodrigo de Illescas
Francisco Sánchez

Francisco Sánchez

?

Alonso Pérez

Alonso Pérez

Alonso Pérez

Manuel Gómez

Manuel Gómez

Manuel Gómez

Manuel Gómez

Manuel Gómez

?

?

?

?

$?$

Gines de Carrión

Ginés de Carrión

Juan Enríquez

Juan Enriquez

?

Cristóbal Rodríguez

Juan Enríquez

Cristóbal Rodríguez

Cristóbal Rodríguez

Cristóbal Rodríguez

Cristóbal Rodríguez ?

Juan Enriquez

Juan Enríquez

Juan Enríquez

Juan Enríquez

Juan Enríquez

Juan Enríquez

Domingo Alonso

Domingo Alonso

16
$180 ?$
$200 ?$

$\begin{array}{rl}19 & \text { TF } \\ 140 & \text { TF } \\ 40 & \text { TF } \\ 60 & \text { NE } \\ 60 & \text { NE } \\ 10 & \text { NE } \\ 8 & \text { NE } \\ 12 & \text { NE } \\ 200 & ? \\ 46 & ? \\ 21 & ? \\ 75 & ? \\ 4 & ? \\ 4 & ? \\ 20 & ? \\ 4 & \text { NE } \\ 2 & \text { NE } \\ 2 & \text { NE } \\ 18 & \text { NE } \\ 2 & \text { NE } \\ 3 & \text { NE } \\ 15 & \text { NE }\end{array}$

$\begin{array}{rl}49 & \mathrm{NE} \\ 48 & \mathrm{NE} \\ 200 & ? \\ 2 & \mathrm{NE} \\ 135 & \mathrm{NE} \\ 2 & \mathrm{NE} \\ 2 & \mathrm{NE} \\ 1 & \mathrm{NE} \\ 2 & \mathrm{NE} \\ 8 & \mathrm{TF} \\ 12 & \mathrm{TF}\end{array}$




Fecha Asentista $\quad$ Maestre $\begin{gathered}\mathrm{N}^{0} \text { de Destino } \\ \text { esclavos }\end{gathered}$

$\begin{array}{ll}\text { 3-IX } & \text { Mariscal Diego Caballero } \\ \text { 3-IX } & \text { Álvaro Caballero } \\ \text { 4-XI } & \text { Alonso Martínez } \\ \text { 12-IX } & \text { Diego Caballero } \\ \text { 13-XI } & \text { Francisco Ponce } \\ \text { 13-XI } & \text { Juan Ambrosio Graso } \\ \text { 14-XI } & \text { Herederos de Hdo. de Jerez } \\ \text { 14-XI } & \text { Alonso García } \\ \text { 10-XII } & \text { Nicolao de Mesina } \\ \text { 11-XII } & \text { Pedro de Galarza } \\ \text { 11-XII } & \text { Mariscal Diego Caballero } \\ \text { 11-XII } & \text { Juan Ruiz de Munzaraz } \\ \text { 12-XII } & \text { Gregorio de Ayala } \\ \text { 12-XII } & \text { Francisco de Vañuelos } \\ \text { 12-XII } & \text { Andrés Gutiérrez } \\ \text { 12-XII } & \text { Juan Caballero } \\ \text { 13-XII } & \text { Gaspar de Torres } \\ \text { 15-XII } & \text { Juan de Oña } \\ \text { 15-XII } & \text { Juan de Lizarazo } \\ \text { 15-XII } & \text { Andrés Gutiérrez }\end{array}$

$\begin{array}{lrl}\text { Domingo Alonso } & 49 & \text { TF } \\ \text { Domingo Alonso } & 10 & \text { TF } \\ \text { Pero López } & 3 & ? \\ \text { ? } & 30 & ? \\ \text { Diego de Quesada } & 3 & \text { TF } \\ \text { Diego de Quesada } & 10 & \text { TF } \\ \text { Diego de Quesada } & 145 & \text { TF } \\ \text { Diego de Quesada } & 2 & \text { TF } \\ \text { Manuel Martín } & 1 & \text { TF ? } \\ \text { Manuel Martín } & 10 & \text { TF ? } \\ \text { Manuel Martín } & 19 & \text { TF? } \\ \text { Manuel Martín } & 1 & \text { TF ? } \\ \text { Manuel Martín } & 45 & \text { TF ? } \\ \text { Manuel Martín } & 4 & \text { TF? } \\ \text { Manuel Martín } & 5 & \text { TF ? } \\ \text { Manuel Martín } & 2 & \text { TF ? } \\ \text { Manuel Martín } & 20 & \text { TF ? } \\ \text { Manuel Martín } & 4 & \text { TF ? } \\ \text { Manuel Martín } & 2 & \text { TF ? } \\ \text { Manuel Martín } & 7 & \text { TF ? }\end{array}$

$\begin{array}{ll}\text { 4-I } & \text { Juan García } \\ \text { 4-I } & \text { G. Fernández de Oviedo } \\ \text { 4-II } & \text { Gaspar de Avila } \\ \text { 8-II } & \text { Rodrigo Pérez } \\ \text { 7-II } & \text { Cristóbal Guillén } \\ \text { 7-II } & \text { Gaspar y Gonzalo Jorge } \\ \text { 7-II } & \text { PJ Leardo y B. de Brine } \\ \text { 7-II } & \text { Gaspar de Ávila } \\ \text { 11-II } & \text { Bartolomé de Vizcarra } \\ \text { 11-II } & \text { Juan de Orduña } \\ \text { 12-II } & \text { Bartolomé de Vizcarra } \\ \text { 12-II } & \text { J. Núñez y hered. de H Jerez } \\ \text { 13-II } & \text { J. Medina de Villavicencio } \\ \text { 15-II } & \text { Asencio de Alcola } \\ \text { 15-II } & \text { Asencio de Alcola } \\ \text { 16-II } & \text { Pedro de Andonaegui } \\ \text { 22-II } & \text { Gaspar Jorge y Juan García } \\ \text { 22-II } & \text { Antonio Corzo }\end{array}$

$\begin{array}{lrl}\text { Gonzalo Vaez } & 115 & \text { LE } \\ \text { Gonzalo Vaez } & 25 & \text { LE } \\ \text { Juan de Palma } & 3 & \mathrm{NE} \\ \text { Juan de Palma } & 28 & \mathrm{NE} \\ \text { P. de Andonaegui } & 1 & \mathrm{H} \\ \text { Juan de Palma } & 140 & \mathrm{NE} \\ \text { Juan de Palma } & 6 & \mathrm{NE} \\ \text { Juan de Palma } & 10 & \mathrm{NE} \\ \text { Fco. de Almansa } & 10 & \mathrm{NE} \\ \text { Fco. de Almansa } & 8 & \mathrm{NE} \\ \text { P. de Andonaegui } & 5 & \mathrm{H} \\ \text { Fco. de Almansa } & 150 & \mathrm{NE} \\ \text { P. de Andonaegui } & 12 & \mathrm{H} \\ \text { P. de Andonaegui } & 60 & \mathrm{H} . \\ \text { P. de Andonaegui } & 40 & \mathrm{H} \\ \text { P. de Andonaegui } & 10 & \mathrm{H} . \\ \text { Sebastián Ramos } & 100 & \mathrm{LE} \\ \text { Scbastián Ramos } & 14 & \mathrm{LE}\end{array}$




Fecha Asentista $\quad$ Maestre $\begin{gathered}\mathrm{N}^{9} \text { de Destino } \\ \text { esclavos }\end{gathered}$

22-II Antonio Corzo

2-III Francisco Rucho

2-III Francisco Núñez Pérez

2-III Francisco Núñez Pérez

2-III Diego Alemán

2-III Francisco Núñez Pérez

2-III Diego Alemán

4-III Francisco Núñez Pérez

4-III Rod. Álvarez y P. Mollinedo

12-III Jurado Gaspar de Torres

2-V Rodrigo Bazo

2-V Rodrigo Bazo

2-V Rodrigo Bazo

4-V Hernando Blas

4-V Rodrigo Bazo

6-VI Francisco de Noya

6-VI Francisco Pérez

22-VI Nicolao Blanco Genoves

26-VI Gerónimo Castaño

3-VIII Gonzalo y Gaspar Jorge

3-VIII Gonzalo y Gaspar Jorge

2-VIII Gonzalo y Gaspar Jorge

3-VIII Vicente Martín

3-VIII Gonzalo y Gaspar Jorge

14-VIII Juan Galbarro

13-IX Juan Galbarro

14-IX Juan Galbarro

9-X Francisco Núñez Pérez

9-X Francisco Núñez Pérez

9-X Francisco Núñez Pérez.

10-X Francisco Núñez Pérez

11-X Ruy Díaz de Gibraleón

11-X Jurado J. de la Barrera

26-X Jurado J. de la Barrera

26-X Alonso de León

29-X Jurado J. de la Barrera

29-X Jurado J. de la Barrera

29-X Jurado J. de la Barrera

5-XI R. Bazo y B. de Jerez

6-XI Rodrigo Bazo y B. de Jerez
Sebastián Ramos

A. Pérez Maldonado

Alonso de Campos

Alonso de Campos

Alonso de Campos

Alonso de Campos

Alonso de Campos

Alonso de Campos

Martín Pérez

A. Pérez Maldonado

Pedro Carrasco

Pedro Carrasco

Pedro Carrasco

Pedro Carrasco

Pedro Carrasco

Gaspar de Acevedo

Gaspar de Acevedo

Gaspar de Acevedo

Gaspar de Acevedo

Cr. de Guadalupe

Cr. de Guadalupe

Cr. de Guadalupe

Cr. de Guadalupe

Cr. de Guadalupe

Bart. de Herrada

Bart. de Herrada

Bart. de Herrada

Fco. de Mena

Fco. de Mena

Fco. de Mena

Fco. de Mena

Fco. de Mena

Fco. de Mena

Juan González

Juan González

Juan González

Juan González

Juan González.

Álv. de Colondres

Álv. de Colondres
36

LE

15 ?

40 NE

3 NE

$24 \quad \mathrm{NE}$

141 NE

$10 \quad \mathrm{NE}$

7 NE

100 ?

$140 \quad \mathrm{NE}$

5 ND

8 ND

100 ND

4 ND

7 ND

3 NE

2 NE

4 NE

190 NE

43 NE

14 NE

40 NE

6 NE

70 NE

45 LE

43 LE

50 LE

135 TF

5 TF

1 TF

8 TF

50 TF

14 TF

50 NE

50 NE

5 NE

$14 \quad \mathrm{NE}$

9 NE

$110 \mathrm{TF}$

2 TF

R. I., 1994, n² 201 


\begin{tabular}{|c|c|c|c|c|}
\hline Fecha & Asentista & Maestre & $\begin{array}{l}\mathrm{N}^{9} \mathrm{de} \\
\text { esclavos }\end{array}$ & Destino \\
\hline
\end{tabular}

$\begin{array}{ll}\text { 6-XI } & \text { Rodrigo Bazo y B. de Jerez } \\ \text { 6-XI } & \text { Alonso Rodríguez } \\ \text { 6-XI } & \text { Alonso Martínez } \\ \text { 6-XI } & \text { Alonso Martinez } \\ \text { 6-XI } & \text { Alonso Núnez } \\ \text { 6-XI } & \text { Rodrigo Bazo } \\ \text { 6-XI } & \text { Alonso Martinez } \\ \text { 7-XI } & \text { Jurado Gaspar de Torres } \\ \text { 7-XI } & \text { Jurado Gaspar de Torres } \\ \text { 7-XI } & \text { Alonso Martinez } \\ \text { 7-XI } & \text { Juan Ramondo } \\ \text { 8-XI } & \text { Jurado J. de la Barrera } \\ \text { 18-XI } & \text { Herederos de H. de Jerez } \\ \text { 18-XI } & \text { Herederos de H. de Jerez } \\ \text { 18-XI } & \text { Jurado Gaspar de Torres } \\ \text { 18-XI } & \text { Gonzalo y Gaspar Jorge } \\ \text { 18-XI } & \text { Domingo de Azpeitia }\end{array}$

14-XII Alonso Martínez

14-XII Alonso Martínez

14-XII Alonso Martínez

14-XII Alonso Martínez

30-XII Fray Juan de Barrios

16-XII Alonso Martínez

16-XII Alonso Martínez

16-XII Alonso Martínez

$\begin{array}{lrl}\text { Álv. de Colondres } & 3 & \text { TF } \\ \text { Alv. de Colondres } & 3 & \text { TF } \\ \text { Francisco Ruiz } & 25 & \text { ND ? } \\ \text { Francisco Ruiz } & 15 & \text { ND ? } \\ \text { Francisco Ruiz } & 50 & \text { ND ? } \\ \text { Álv. de Colondres } & 2 & \text { TF } \\ \text { Francisco Ruiz } & 100 & \text { ND ? } \\ \text { ? } & 101 & ? \\ \text { Antonio Carnero } & 100 & ? \\ \text { Francisco Ruiz } & 6 & \text { ND ? } \\ \text { Diego de Quesada } & 2 & \text { TF } \\ \text { Álv. de Colondres } & 20 & \text { TF } \\ \text { Diego de Quesada? } & 160 & \text { TF } \\ \text { Diego de Quesada } & 140 & \text { TF } \\ \text { Diego de Quesada } & 30 & \text { TF } \\ \text { Juan B. Gallegos } & 18 & \text { TF } \\ \text { Domingo de Azpei- } & & \\ \text { tia } & 4 & ? \\ \text { Crist de Angulo } & 20 & ? \\ \text { Crist. de Angulo } & 75 & ? \\ \text { Crist. de Angulo } & 75 & ? \\ \text { Crist. de Angulo } & 10 & ? \\ \text { J. Sánchez de Viz- } & & \\ \text { caya } & 3 & \text { RP } \\ \text { Crist. de Angulo } & 15 & ? \\ \text { Crist. de Angulo } & 5 & ? \\ \text { Crist. de Angulo } & 10 & ?\end{array}$

1550

$\begin{array}{lrr}\text { Domingo Alonso } & 4 & \mathrm{NE} \\ \text { Domingo Alonso } & 135 & \mathrm{NE} \\ \text { Domingo Alonso } & 10 & \mathrm{NE} \\ \text { Domingo Alonso } & 10 & \mathrm{NE} \\ \text { Alonso Granillo } & 100 & \mathrm{NE} \\ \text { A. Pérez Gallego } & 100 & \mathrm{NE} \\ \text { Alonso Granillo } & 50 & \mathrm{NE} \\ \text { A. Pérez Gallego } & 20 & \mathrm{NE} \\ \text { A. Pérez Gallego } & 27 & \mathrm{NE} \\ \text { A. Pérez Granillo } & 18 & \mathrm{NE} \\ \text { Gaspar Salvago } & 197 & \mathrm{PR}\end{array}$




\begin{tabular}{|c|c|c|c|c|}
\hline Fecha & Asentista & Maestre & $\begin{array}{c}\mathrm{N}^{\mathrm{Q}} \mathrm{de} \\
\text { esclavos }\end{array}$ & Destino \\
\hline
\end{tabular}

$\begin{array}{ll}\text { 23-V } & \text { Diego de Villalán } \\ \text { 23-V } & \text { Carlos de Negrón } \\ \text { 23-V } & \text { Antonio Fresco } \\ \text { 28-V } & \text { G. Ruiz de Huelva } \\ \text { 29-V } & \text { Alonso Martínez } \\ \text { 29-V } & \text { Alonso Martínez } \\ \text { 29-V } & \text { Alonso Martínez } \\ \text { 29-V } & \text { Alonso Martínez } \\ \text { 29-V } & \text { Alonso Martínez } \\ \text { 12-VIII } & \text { Fco. Núñez Pérez } \\ \text { 12-VIII } & \text { Fco. Núñez Pérez } \\ \text { 12-IX } & \text { Fco. Núñez Pérez } \\ \text { 12-IX } & \text { Fco. Núñez Pérez } \\ \text { 12-IX } & \text { Fco. Núñez Pérez } \\ \text { 13-X } & \text { Alonso Martínez } \\ \text { 13-X } & \text { Fco. Núñez Pérez } \\ \text { 13-X } & \text { Antón de Segovia } \\ \text { 13-X } & \text { Gaspar de los Reyes } \\ \text { 12-XI } & \text { Gonzalo Jorge } \\ \text { 12-XI } & \text { Antón Gómez } \\ \text { 12-XI } & \text { Fco. Núñez Pérez } \\ \text { 12-XI } & \text { Fco. Núñez Pérez } \\ \text { 12-XI } & \text { Gaspar Jorge } \\ \text { 12-XI } & \text { Gonzalo y Gaspar Jorge } \\ \text { 13-XI } & \text { J. Núñez y hered. de H. Jerez } \\ & \end{array}$

$\begin{array}{lrl}\text { A. Pérez Gallego } & 23 & \mathrm{NE} \\ \text { Jácome Dominguez } & 120 & ? \\ \text { Jácome Dominguez } & 95 & ? \\ \text { Diego de Luna } & 80 & \mathrm{NE} \\ \text { Diego de Luna } & 65 & \mathrm{NE} \\ \text { Diego de Luna } & 15 & \mathrm{NE} \\ \text { Diego de Luna } & 15 & \mathrm{NE} \\ \text { Diego de Luna } & 3 & \mathrm{NE} \\ \text { Diego de Luna } & 2 & \mathrm{NE} \\ \text { Miguel de Oquendo } & 34 & \mathrm{NE} \\ \text { Miguel de Oquendo } & 140 & \mathrm{NE} \\ \text { Miguel de Oquendo } & 40 & \mathrm{NE} \\ \text { Miguel de Oquendo } & 20 & \mathrm{NE} \\ \text { Miguel de Oquendo } & 5 & \mathrm{NE} \\ \text { Martín Garrucho } & 150 & \mathrm{NE} ? \\ \text { Martín Garrucho } & 4 & \mathrm{NE} ? \\ \text { Martín Garrucho } & 21 & \mathrm{NE} ? \\ \text { Martín Garrrucho } & 10 & \mathrm{NE} ? \\ \text { Antonio Gómez } & 48 & \mathrm{NE} ? \\ \text { Antonio Gómez } & 2 & \mathrm{NE} ? \\ \text { Toribio García } & 4 & \mathrm{NE} \\ \text { Toribio García } & 3 & \mathrm{NE} \\ \text { Antón Gómez } & 86 & \mathrm{ND} \text { ? } \\ \text { Toribio Garcia } & 12 & \mathrm{NE} \\ \text { Toribio García } & 200 & \mathrm{NE}\end{array}$

Fuente:

Archivo General de Simancas. Consejo y Junta de Hacienda, 23, fols. 388-488. 